\title{
Optical Coherence Tomography Changes in Children with Unilateral Amblyopia
}

\author{
Farid Mohammed Wagdy, Adel Galal Zaky, Sara Fathallah Mohammed EI Shafey \\ Ophthalmology department, Faculty of Medicine, Menoufia University
}

Corresponding author: Prof. Dr. Farid Mohammed Wagdy, Ophthalmology department, Faculty of Medicine, Menoufia University, Egypt. Tel: 00201001227987; Email: fariedwagdy1976@gmail.com.

Received: 5-8-2021, Accepted: 1-10-2021, Published online:16-12-2021

EJO(MOC) 2021;4:198-205.

Running title: OCT changes in children with unilateral amblyopia.

\section{ABSTRACT}

Purpose: Amblyopia is one of the most common causes of visual loss in children affecting $0.2 \%$ to $1.1 \%$ of school going children. A difference in refractive error between the two eyes (anisometropia) is a common cause of amblyopia, being present as the only identifiable amblyogenic factor in $37 \%$ of cases and present concomitantly with strabismus in an additional $24 \%$ of clinical populations.

Objective: The aim of this study is to determine the changes in retinal nerve fiber layer thickness and macular thickness in different types of amblyopia using optical coherence tomography.

Patients and methods: This cross-sectional study was conducted at Ophthalmology Departments of Menoufia University Hospitals. It was carried out on 144 eyes of 72 patients with unilateral amblyopia divided as strabismic amblyopic group ( $\mathrm{n}=$ $24)$, derivational amblyopic group $(n=11)$ and anisometropic amblyopic group $(n=37)$.

Results: there was no statistically significant differences in the mean of RNFL thickness and also mean of central \& average macular thickness in amblyopic eye compared to fellow eye in all groups expect in (Deprivational group) there was highly statistically significant decrease in the mean of inferior RNFL thickness \& highly statistically significant increase in the mean of central macular thickness in amblyopic eye compared to fellow eye.

Conclusion: These findings suggested that there no changes in retinal nerve fiber layer thickness and macular thickness in different types of amblyopia using optical coherence tomography except in deprivational group These findings suggested that there no changes in retinal nerve fiber layer thickness and macular thickness in different types of amblyopia using optical coherence tomography except in deprivational group. This may be a diagnostic tool to differentiate between deprivation amblyopia and different types of amblyopia. Also this may encourage further neurological investigation $\mathrm{s}$ to know pathophysiological neurological changes that occur which may have its influence for a future therapy of deprivation amblyopia.

Keywords: Optical coherence tomography, amblyopic children.

\section{INTRODUCTION}

Amblyopia can be defined as reduced best corrected visual acuity (BCVA) in one or both eyes caused by abnormal visual experience during visual development. It may be caused by sensory deprivation, image blur from refractive errors, strabismus, or combination of these factors ${ }^{1}$.

The three main types of amblyopia are: strabismic, anisometropic and from vision deprivation. It has been thought

Egyptian Journal of Ophthalmology, a publication of Mansoura Ophthalmic Center.

Address: Mansoura Ophthalmic Center, Mansoura University, Mansoura, Egypt.

Tel. 0020502202064. Fax. 0020502202060.

E-mail: ejo@mans.edu.eg 
that only functional changes in visual cortex and lateral geniculate body can be observed in patient with amblyopia.

The matter of retinal changes in amblyopia is still inconclusive and under investigation ${ }^{2}$.

Amblyopia usually associated with changes in the distribution of ocular dominance patterns in the visual cortex, cell shrinkage in the lateral geniculate body, and optic nerve hypoplasia ${ }^{3}$.

Retinal nerve fiber layer (RNFL) is considered a very sensitive indicator of early glaucoma preceding other structural and functional changes of glaucomatous damage ${ }^{4}$. It remains a questionable topic in amblyopia study Does RNFL thickness differ in amblyopic eyes or not? This could be possible through comparing RNFL thickness in normal fellow and amblyopic eyes in order to make more clinically useful normative databases of RNFL thickness which help early diagnosis and monitoring of glaucoma and optic nerve disorders ${ }^{5}$. Many techniques can be used to assess retinal nerve fiber layer thickness such as scanning laser polarimetry (SLP), red free ophthalmoscopy and optical coherence tomography $(\mathrm{OCT})^{\mathbf{6}}$.

Optical coherence tomography (OCT) is a non-contact, noninvasive method of using low-coherence interferometry to detect the echo magnitude and time delay of back scattered light reflected off an object of interest leading to exploration of microstructure within turbid media. OCT is similar to US but using light instead of sound and measuring optical rather than acoustic or radio wave and hence the term (optical), ${ }^{7}$ OCT provides a cross sectional image of the anterior eye and retina and hence the term tomography (imaging by sectioning).

OCT image in vivo has a high resolution similar to a histological section by light microscopy in vitro ${ }^{8}$. OCT imaging is based on correlation techniques that compare the back scattered or back reflected light signal to reference light traveling a known path length (direct detection of light echoes is not possible because the speed of light is very high), ${ }^{\mathbf{9}}$.

\section{Aim of the Work}

The aim of this study is to determine the changes in retinal nerve fiber layer thickness and macular thickness in different types of amblyopia using optical coherence tomography.

\section{PATIENTS AND METHODS}

I. Patients: This cross-sectional study was conducted at Ophthalmology Departments of Menoufia University Hospitals. It was carried out on 144 eyes of 72 patients with unilateral amblyopia.

Those patients were classified according to their amblyogenic factor into three groups:

- Group I. Strabismic amblyopic group $(\mathrm{n}=24)$.

- Group II. Deprivational amblyopic group $(n=11)$.

- Group III. Anisometropic amblyopic group $(\mathrm{n}=37)$

And then anisometropia amblyopic group (III) was further classified according to their refractive power into three subgroups:

- Sub group a) Anisoastigmatic subgroup (n=9).

- Sub group b) Anisohypermetroic subgroup ( $\mathrm{n}=15)$.

- Sub group c) Anisomyopic subgroup (N=13).

\section{Ethical consideration and written informed consent:}

An approval of the study was obtained from Menoufia University Academic Ethical Committee. Every patient signed an informed written consent for acceptance of the operation.

Inclusion criteria: included cooperative patients aged 7 to 18 years old with different types of amblyopia, and in anisometropic group refraction equals or less than -8 diopters myopic, +6 hyperopic and 4 diopters astigmatism.

Exclusion criteria: The following patients were excluded from the study:

1. Uncooperative patients.

2. Mixed amblyopia patients.

3. Patients with severe amblyopia with poor or eccentric fixation.

4. Patients with any structural abnormalities of the eye, either congenital or acquired.

5. Mental retarded patients.

II. All individuals included in this study were subjected to the following:

1-Full history taking: Complete personal history taking including age, sex and residence. History of previous ocular 
operation, history of wearing glasses, history of previous occlusion therapy, or amblyopia therapy in general.

\section{2- Comprehensive ophthalmological examination:}

Unaided visual acuity and best corrected visual assessment by landot (broken ring "c") chart, then the visual acuity will be converted into logarithm of minimum angle of resolution (log MAR) for easier statistical analysis. Pupillary dilatation and cycloplejia will perform with cyclopentolate $\mathrm{Hcl} 0.1 \%$.

- Fundus examination: using either slit lamp bio microscopy, using non-contact Volk 90 lens, indirect or direct ophthalmoscopy.

- Slit lamp examination for anterior segment examination to exclude any anterior segment pathology.

- Ocular tension will measure by Goldmann applanation tonometer or schiotz indentation tonometer.

- Ocular motility assessment. Cover-uncover test.

- Optical coherence tomography imaging.

The peripapillary RNFL thickness for the four quadrants (superior, inferior, nasal, temporal) and the central macular thickness were measured by using Spectralis ${ }^{\circledR}$ spectral domain optical coherence tomography (Heidelberg engineering, Heidelberg, Germany) (Specralis software version 4.0).

The CMT is defined as the central circle of $1 \mathrm{~mm}$ diameter in the macular image was measured by fast macular thickness protocol, $6 \mathrm{~mm}$ lines in a radial spoke pattern obtained in a continuous automated sequence. Internal fixation was used for all scans. Peripapillary RNFL thickness was measured using fast RNFL thickness (3.4 mm diameter) scan the central circle of the RNFL scan which is called "global" and the inferior, temporal, superior, and nasal thickness was used to calculate the difference between the ametropic and the normal eyes. The average of three sets of measurements were collected, calculated and tabulated then statistically analyzed and the values of the two eyes.

\section{Statistical analysis}

The data collected were tabulated $\&$ analyzed by SPSS (statistical package for the social science software) statistical package version 26 on IBM compatible computer. Quantitative data were expressed as mean \& standard deviation $(\mathrm{X}+\mathrm{SD})$ and analyzed by applying studentt-test for comparison of two groups of normally distributed variables and Mann-Whiteny test for nonnormally distributed ones. Chi-Square test was used to compare categorical variables. $\mathrm{P}$ value $<0.05$ was considered statistically significant in all these tests.

\section{RESULTS}

72 patients (144 eyes) are included in this study, age of the studied groups was 7-18 years, 50\% for each amblyopic and fellow eyes (72 eyes for other one), patients were demographically distributed into 38 male and 34 female (Table $1)$.

Table (1): Demographic data of the patient

\begin{tabular}{ll}
\hline Demographic & $\mathbf{N}=\mathbf{7 2}$ \\
\hline Age, range (mean \pm SD), years & $8-18(13.2 \pm 3.19)$ \\
Sex & 34 females \\
& 38 males \\
\hline
\end{tabular}

Regarding peripapillary RNFL, there was highly statistically significant increase in the mean of Nasal RNFL thicken $(89.56 \pm 32.53)$ in amblyopic eye compared to fellow eye $(75.21 \pm 16.59)$ but there were no statistically significant differences between two eyes as regard the mean of superior, inferior and temporal RNFL thickness (Table 2).

Table (2): RNFL thickness of all study group

\begin{tabular}{lcccc}
\hline & $\begin{array}{c}\text { Amblyopic eye } \\
(\mathbf{n}=\mathbf{7 2})\end{array}$ & $\begin{array}{c}\text { Fellow eye } \\
(\mathbf{n}=\mathbf{7 2})\end{array}$ & t. test & P value \\
\hline RNFL thickness & & & & \\
Superior & $132.28 \pm 31.11$ & $130.57 \pm 20.45$ & 0.39 & 0.70 \\
Inferior & $126.13 \pm 24.40$ & $123.99 \pm 21.20$ & 0.56 & 0.58 \\
Nasal & $89.56 \pm 32.53$ & $75.21 \pm 16.59$ & 3.33 & $0.001 * *$ \\
Temporal & $77.53 \pm 17.53$ & $83.38 \pm 25.09$ & -1.62 & 0.11 \\
\hline
\end{tabular}


Also, according to amblyogenic factor classification in amblyopic eyes, there was highly statistically significant decrease in the mean of inferior RNFL thickness in amblyopic eye (deprivational group), (109.33 \pm 9.97$)$

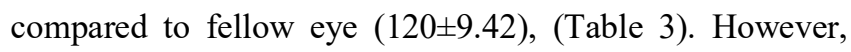
there was statistically significant decrease in the mean of superior RNFL (112.67 \pm 25.52$)$, inferior RNFL $(123.11 \pm 27.45)$ and nasal RNFL $(72.56 \pm 12.60)$ with statistically significant increase in the mean of temporal RNFL (76.89 \pm 11.65$)$ in amblyopic eyes (anisoastigmatic subgroup) compared to fellow eyes, while there was statistically significant increase in the mean of superior RNFL (148.60 \pm 45.02$)$ and nasal RNFL (106.87 \pm 50.46$)$ in amblyopic eyes (anisohypermetroic subgroup) compared to fellow eyes, (Table 4).

Table (3): RNFL thickness in Deprivational group

\begin{tabular}{lcccc}
\hline \multicolumn{1}{c}{ RNFL thickness } & $\begin{array}{c}\text { Amblyopic eye } \\
(\mathbf{n = 1 1 )}\end{array}$ & $\begin{array}{c}\text { Fellow eye } \\
(\mathbf{n}=\mathbf{1 1})\end{array}$ & F test & P-Value \\
\hline Superior & $130.67 \pm 5.39$ & $129 \pm 3.58$ & 0.925 & 0.397 \\
Inferior & $109.33 \pm 9.97$ & $120 \pm 9.42$ & -6.325 & $0.001 * *$ \\
Nasal & $64.00 \pm 12.03$ & $78.33 \pm 22.65$ & -2.461 & 0.057 \\
Temporal & $100.67 \pm 15.34$ & $83.00 \pm 11.63$ & 2.462 & 0.057 \\
\hline
\end{tabular}

Table (4): RNFL thickness in Anisometropic group

\begin{tabular}{|c|c|c|c|c|c|}
\hline & RNFL thickness & $\begin{array}{c}\text { Amblyopic eye } \\
(n=37)\end{array}$ & $\begin{array}{c}\text { Fellow eye } \\
\quad(n=37)\end{array}$ & T- test & P-Value \\
\hline \multirow{4}{*}{ Superior } & Total & $127.00 \pm 39.29$ & $122.47 \pm 21.22$ & 0.757 & 0.460 \\
\hline & Anisoastigmatic amblyopia & $112.67 \pm 25.52$ & $124.80 \pm 20.91$ & 3.753 & $0.020 *$ \\
\hline & Anisohypermetropic amblyopia & $148.60 \pm 45.02$ & $126.33 \pm 23.77$ & 1.45 & $0.018^{*}$ \\
\hline & Anisomyopic amblyopia & $105.15 \pm 23.09$ & $104.00 \pm 0.00$ & 1.554 & 0.364 \\
\hline \multirow{4}{*}{ Inferior } & Total & $127.65 \pm 31.90$ & $129.94 \pm 23.85$ & -0.815 & 0.427 \\
\hline & Anisoastigmatic amblyopia & $123.11 \pm 27.45$ & $128.40 \pm 21.33$ & 3.439 & $0.026^{*}$ \\
\hline & Anisohypermetropic amblyopia & $139.67 \pm 36.26$ & $135.00 \pm 27.73$ & 2.14 & 0.06 \\
\hline & Anisomyopic amblyopia & $116.54 \pm 25.76$ & $109.00 \pm 0.00$ & 2.071 & 0.286 \\
\hline \multirow{4}{*}{ Nasal } & Total & $86.53 \pm 37.82$ & $77.88 \pm 14.26$ & 0.921 & 0.371 \\
\hline & Anisoastigmatic amblyopia & $72.56 \pm 12.60$ & $81.60 \pm 17.90$ & 5.160 & $0.017^{*}$ \\
\hline & Anisohypermetropic amblyopia & $106.87 \pm 50.46$ & $76.11 \pm 15.30$ & 2.06 & $0.004^{*}$ \\
\hline & Anisomyopic amblyopia & $84.85 \pm 35.85$ & $77.00 \pm 0.00$ & 1.573 & 0.361 \\
\hline \multirow{4}{*}{ Temporal } & Total & $71.47 \pm 9.45$ & $69.94 \pm 31.34$ & 0.205 & 0.841 \\
\hline & Anisoastigmatic amblyopia & $76.89 \pm 11.65$ & $63.00 \pm 9.92$ & 3.696 & $0.021 *$ \\
\hline & Anisohypermetropic amblyopia & $71.87 \pm 9.37$ & $78.11 \pm 41.78$ & 3.84 & 0.051 \\
\hline & Anisomyopic amblyopia & $66.77 \pm 14.86$ & $58.00 \pm 0.00$ & 2.019 & 0.293 \\
\hline
\end{tabular}

Regarding, refractive power classification of anisometropic group, there was no statistically significant differences in the mean of central and average macular thickness in amblyopic eye compared to fellow eye in all subgroups except in anisoastigmatic subgroup, there was statistically highly significant increase in average macular thickness (296.78 \pm 15.99$)$ compared to fellow eye (283.40 \pm 48.84$)$, (Table 5). 
Table (5): Macular thickness and volume in Anisometropic group

\begin{tabular}{|c|c|c|c|c|c|}
\hline \multicolumn{2}{|c|}{ Anisometropic group } & $\begin{array}{c}\text { Amblyopic eye } \\
(\mathrm{n}=37)\end{array}$ & $\begin{array}{c}\text { Fellow eye } \\
(n=37)\end{array}$ & T- test & P-Value \\
\hline \multirow{4}{*}{$\begin{array}{l}\text { Central Macular } \\
\text { thickness }\end{array}$} & Total & $265.06 \pm 10.25$ & $261.41 \pm 14.11$ & 1.173 & 0.258 \\
\hline & Anisoastigmatic amblyopia & $268.33 \pm 14.14$ & $267.20 \pm 9.44$ & 1.628 & 0.179 \\
\hline & $\begin{array}{l}\text { Anisohypermetropic } \\
\text { amblyopia }\end{array}$ & $258.93 \pm 12.27$ & $256.78 \pm 14.88$ & 2.31 & 0.05 \\
\hline & Anisomyopic amblyopia & $257.23 \pm 29.98$ & $276.00 \pm 0.00$ & 0.388 & 0.765 \\
\hline \multirow{4}{*}{$\begin{array}{l}\text { Average macular } \\
\text { volume }\end{array}$} & Total & $8.95 \pm 0.29$ & $8.48 \pm 0.66$ & 3.163 & $0.006 * *$ \\
\hline & Anisoastigmatic amblyopia & $8.61 \pm 0.63$ & $8.40 \pm 0.49$ & 3.426 & 0.262 \\
\hline & $\begin{array}{l}\text { Anisohypermetropic } \\
\text { amblyopia }\end{array}$ & $8.99 \pm 0.43$ & $8.38 \pm 0.66$ & 2.642 & 0.133 \\
\hline & Anisomyopic amblyopia & $8.15 \pm 1.26$ & $8.37 \pm 0.00$ & 3.812 & 0.163 \\
\hline \multirow{4}{*}{$\begin{array}{l}\text { Average macular } \\
\text { thickness }\end{array}$} & Total & $294.29 \pm 33.08$ & $291.88 \pm 29.28$ & 0.365 & 0.720 \\
\hline & Anisoastigmatic amblyopia & $296.78 \pm 15.99$ & $283.40 \pm 48.84$ & 32.157 & $0.022 *$ \\
\hline & $\begin{array}{l}\text { Anisohypermetropic } \\
\text { amblyopia }\end{array}$ & $291.20 \pm 34.82$ & $299.44 \pm 18.99$ & 11.45 & 0.053 \\
\hline & Anisomyopic amblyopia & $280.92 \pm 48.04$ & $289.00 \pm 0.00$ & 16.32 & 0.073 \\
\hline
\end{tabular}

\section{DISCUSSION}

OCT achieves reliable cross-sectional stratification of the retinal layers. It enabled detailed exploration for pathological changes in the retina. Also, it permitted meticulous results and analysis for peripapillary nerve fiber layer ${ }^{2}$. In our study, we analyzed the thickness of peripapillary RNFL and macula thickness and volume comparing between the amblyopic and fellow eye for 72 patients (144 eyes) by SD- OCT.

Regarding peripapillary RNFL, there was highly statistically significant increase in the mean of Nasal RNFL thicken (89.56 \pm 32.53$)$ in amblyopic eye compared to fellow eye $(75.21 \pm 16.59)$ but there were no statistically significant differences between two eyes as regard the mean of superior, inferior and temporal RNFL thickness. Also, according to Amblyogenic factor classification in amblyopic eyes, there was no statistically significant differences in the mean of RNFL thickness in amblyopic eye compared to fellow eye in all groups expect in (deprivational group) there was highly statistically significant decrease in the mean of inferior RNFL thickness in amblyopic eye (109.33 \pm 9.97$)$ compared to fellow eye (120 \pm 9.42$)$
Regarding, refractive power classification of anisometropic group there was no statistically significant difference in amblyopic eyes (anisomyopic subgroup) compared to fellow eyes. However, there was statistically significant decrease in the mean of superior RNFL (112.67 \pm 25.52$)$, inferior RNFL (123.11 \pm 27.45$)$ and nasal RNFL (72.56 \pm 12.60$)$ with statistically significant increase in the mean of temporal RNFL $(76.89 \pm 11.65)$ in amblyopic eyes (anisoastigmatic subgroup) compared to fellow eyes, while there was statistically significant increase in the mean of superior RNFL (148.60 \pm 45.02$)$ and nasal RNFL $(106.87 \pm 50.46)$ in amblyopic eyes (anisohypermetroic subgroup) compared to fellow eyes.

Several previous studies indicated results similar to our; For instance, $\mathrm{Bob}^{11}$, found no significant difference in RNFL thickness in the amblyopic and the fellows' eyes was $(103.4 \pm 12.0)$ and $(103.4 \pm 17.2)$ respectively. This study was limited to anisometropia hyperopic amblyopic children. The conclusion of Repk ${ }^{12}$ and his colleagues reached to similar to the previous results with various types and degrees of refractive errors and a wide age range, not confined to certain 
type of refractive error or children only. They concluded that RNFL thickness was insignificantly\| thicker in hyperopic amblyopic eye. Also, Colen et al., ${ }^{13}$ confirmed their results when they studied strabismic amblyopia in children and also reported no statistically difference in thickness of retinal nerve fiber layer between amblyopic and normal fellow eyes using nerve fiber analyzer.

Several studies were done about amblyopia and give several results in contrast to our study showed no significant results according to anisometropic amblyopic eye and this supported by Altintas et al., ${ }^{14}$, who reported that the RNFL thickness was thicker in amblyopic eyes, but that the difference was not statistically significant. Similar results were reported by Kee et al. ${ }^{15}$ and Firat et al. ${ }^{16}$

A different result with our study as regarding to anisohyperopia Singh et al. ${ }^{17}$ reported that in anisohypermetropia only inferior quadrant RNFL was found to be significantly thicker in eyes with greater refractive error but our study temporal quadrant was the thicker. Other studies measured RNFL thickness in patients with unilateral amblyopia (strabismic and refractive) and found no significant difference between the eyes of strabismic amblyopes; however, they found RNFL to be significantly thicker in refractive amblyopes. They postulated that amblyopic eyes have a thicker retina due to the arrest of the physiological postnatal ganglion cell reduction Yen et al., ${ }^{18}$ With the booming technology, Yoon et al. ${ }^{19}$ studied 31 patients with hyperopic anisometropic amblyopia, they found RNFL thickness was more in amblyopic eyes than in fellows.

Al-Otaibi et al. ${ }^{20}$ enrolled 93 patients in their study; 36 candidates suffering from unilateral strabismic amblyopia, 33 with anisometropic amblyopia and 24 patients were mixed type. They noticed increased circumpapillary retinal nerve fiber layer thickness in all types of amblyopia. researches carried out with $\mathrm{Wu}$ et al. ${ }^{21}$ supported increased RNFL thickness in anisometropic amblyopic eyes than in fellow eyes. According to amblyopic factor classification in amblyopic eyes, there was no statistically significant differences in the mean of central \& average macular thickness in amblyopic eye compared to fellow eye in all groups expect in deprivational group, there was highly statistically significant increase in the mean of central macular thickness in amblyopic eye (270.67 \pm 11.81$)$ compared to fellow eye $(266.67 \pm 11.25)$.

Also, according to refractive power classification of anisometropic group, there was no statistically significant differences in the mean of central and average macular thickness in amblyopic eye compared to fellow eye in all subgroups except in anisoastigmatic subgroup, there was statistically highly significant increase in average macular thickness (296.78 \pm 15.99$)$ compared to fellow eye (283.40 \pm 48.84$)$. Against to our study according to CMTD Demircan et al. ${ }^{21}$ who done observations on CMT in anisohypermetroic subjects and found significantly greater mean central macular thickness in anisometropic amblyopic eyes among participants aged 5 to 12 years, but not in those who were older. To the best of our knowledge there are no studies in the literature on CMT in anisoastigmatism amblyopia. $^{22}$

Another study of KonuralpYakar, et al. ${ }^{23}$ reported no significant difference in central macular thickness, as CMT was (266.9 \pm 23.2$)$ and (263.9 \pm 22.84$)$, while RNFL thickness was $(111.9 \pm 12.9)$ and $(109.7 \pm 9.42)$ in the amblyopic and fellow eyes respectively. Unlike the previous study, this one was limited to anisomertopic hyperopic amblyopic "adults. Additionally, two other studies were limited only to unilateral strabismic amblyopia and ended with the above results reporting no difference between amblyopic and normal fellow eyes as a control group: the study of $\mathrm{Xu}$ et al. ${ }^{24}$ and the second was the study of Reza Zarei et al., which had the same results but with use of scanning polarimetry ${ }^{25}$.

\section{CONCLUSION}

These findings suggested that there no changes in retinal nerve fiber layer thickness and macular thickness in different types of amblyopia using optical coherence tomography except in deprivational group These findings suggested that there no changes in retinal nerve fiber layer thickness and macular thickness in different types of amblyopia using optical coherence tomography except in deprivational group. This may be a diagnostic tool to differentiate between deprivation amblyopia and different types of amblyopia. Also, this may encourage further neurological investigations to know 
pathophysiological neurological changes that occur which may have its influence for a future therapy of deprivation amblyopia.

\section{DATA AVAILABILITY}

All data are included in this article.

\section{ACKNOWLEDGEMENT}

None

\section{Conflict of Interest}

Authors declare no conflicts of interest.

Corresponding author

Correspondence to: Farid Mohammed Wagdy

Email: fariedwagdy1976@gmail.com

\section{Affiliations}

Farid Mohammed Wagdy, Ophthalmology department, Faculty of Medicine, Menoufia University, Egypt.

\section{Ethics declarations}

\section{Conflict of interest}

Farid Mohammed Wagdy, Adel Galal Zaky, Sara Fathallah Mohammed El Shafey, all authors have no conflicts of interest that are directly relevant to the content of this review.

Funding: No sources of funding were used to conduct this review.

Reviewer disclosures: No relevant financial or other relationships to disclose.

Declaration of interest: No financial affiliations or financial involvement with any organization or entity with a financial competing with the subject matter or materials discussed in the review.

\section{REFERENCES}

1. Repka, MX, Kraker RT, Tamkins SM, Suh DW, Sala NA, Beck RW. Retinal nerve fiber layer thickness in amblyopic eyes. AJO 2009;148(1):143-147.

2. Araki S, Miki A, Yamashita T, Goto K, Haruishi K, Ieki Y, Kiryu J. A comparison between amblyopic and fellow eyes in unilateral amblyopica using spectral domain optical coherence tomography, clinical ophthalmology 2014;10:7/OPTH.S69501.

3. Wang BZ, Taranath D. A comparison between the amblyopic eye and normal fellow eye ocular architecture in children with hyperopic anisometropic amblyopia. JAAPOS 2012;16(5):428-30.

4. Sowmya V, Venkataramanan VR, PraSad V. Effect of refractive status and axial length on peripapillary retinal nerve fibre layer thickness: an analysis using 3D OCT. JCDR 2015;9(9):01-04.

5. Öner V, Aykut V, Tas M, Alaks MF, and Iscan Y. Effect of refractive status on peripapillary retinal nerve fibre layer thickness: study by RTVue spectral domain optical coherence tomography. BJO 2013;97(1):75-9.

6. Park KA, Park DY, Oh SY. Analysis of spectral-domain OCT measurement in amblyopia, a pilot study. British journal Ophthalmol. 2011; 95(12):1700-1706.

7. Otani, T, Yamaguchi Y, Kishi S. Improved visualization of Henle fiber layer by changing the measurement beam angle on optical coherence tomography. Retina 2011;31(3):497-501.

8. Yonetsu T, Bouma BE, Kato K, Fujimoto JG, and Jang IK. Optical coherence tomography. Circulation Journal, 2013;77(8): p. 1933-1940.

9. Bressler SB, Edwards AR, Chalam KV, Bressler NM, Glassman AR, Jafee JG. Reproducibility of spectraldomain optical coherence tomography retinal thickness measurements and conversion to equivalent time-domain metrics in diabetic macular edema. JAMA ophthalmology, 2014;132(9): 1113-112.

10. Patel MD, Khushzad F, Moss HE. Comparison of crosssectional optical coherence tomography images of elevated optic nerve heads across acquisition devices and scan protocols. Eye and Vision. 2018 Dec;5(1):1-9.

11. Repka, MX, Kraker RT, Tamkins SM, Suh DW, Sala NA, Beck RW. Retinal nerve fiber layer thickness in amblyopic eyes. AJO 2009;148(1):143-147. 
12. Colen TP, DE Faber. Retinal nerve fiber layer thickness in humenstrabismic amblyopia. Binocul. vis. strabismus $2005 ; 2005(15): 141-146$.

13. Altintas, O., Yüksel, N., Ozkan, B., Çaglar, Y. Thickness of the retinal nerve fiber layer, macular thickness, and macular volume in patients with strabismic amblyopia. Journal of pediatric ophthalmology and strabismus, 2005; 42(4):216.

14. Kee, S. Y., Lee, S. Y., Lee, Y. C. Thicknesses of the fovea and retinal nerve fiber layer in amblyopic and normal eyes in children. Korean Journal of Ophthalmology, 2006; 20(3):177-181.

15. Firat, PG, Ozsoy E, Demirel S, Cumurcu T, Gunduz A. Evaluation of peripapillary retinal nerve fiber layer, macula and ganglion cell thickness in amblyopia using spectral optical coherence tomography. IJO 2013;6(1):90.

16. Singh, N., Rohatgi, J., Gupta, V. P., \& Kumar, V. Measurement of peripapillary retinal nerve fiber layer thickness and macular thickness in anisometropia using spectral domain optical coherence tomography: a prospective study. Clinical Ophthalmology, 2017;11(3), 429-432.

17. Yen MY, Cheng CY, Wang AG. Retinal nerve fiber layer thickness in unilateral amblyopia. IOVS 2004; 45(7):2224-2230.

18. Yoon SW, Park WH, Baek SH, and Kong SM. Thicknesses of macular retinal layer and peripapillary retinal nerve fiber layer in patients with hyperopic anisometropic amblyopia. KJO 2005;19(1):62-67.

19. Alotaibi AG, AL Enazi B. "unilateral amblyopia: optical coherence tomography findings". Saudi journal of ophthalmology 2011;25:405-409.

20. Wu, S. Q., Zhu, L. W., Xu, Q. B., Xu, J. L., \& Zhang, Y. (Macular and peripapillary retinal nerve fiber layer thickness in children with hyperopic anisometropic amblyopia. International journal of ophthalmology, 2013;6(1):85.

21. Demircan S, Gokce G, Yuvaci I, Ataş M, Başkan B, and Zararsiz G, The assessment of anterior and posterior ocular structures in Hyperopic Anisometropic Amblyopia. MessciMonit, 2015;21:1181- 1188.

22. Yakar K, Kan E, Alan A, Alp MH, Ceylan T. Retinal nerve fibre layer and macular thicknesses in adults with hyperopic anisometropic amblyopia. JOO 2015;Doi: $10.1155 / 2015 / 946467$.

23. Xu J, Lu F, Liu W, Zhang F, Chen W, Chen J. Retinal nerve fibre layer thickness and macular thickness in patients with esotropic amblyopia. Clinical and experimental optometry 2013; 96(3):267-271.

24. Zarei R, Anvari F, Abdollahi A, Jabbarvand M, Khademian M, Maleki M. Comparison of Retinal Nerve Fiber Layer Thickness between Amblyopic and Normal Eyes in unilateral strabismic Amblyopia using Scanning Laser polarimetry. IRJO 2009;21(3):17. 